\section{Jejunal feeding: when is it the right thing to do?}

This is a complex topic - straightforward if there is a structural problem which precludes oral or intra gastric feeding, but more complicated in patients with no clear structural abnormality. Paine and colleagues review the issues - what is the goal of therapy, is the patient malnourished, the different causes, the practicalities, the need for an exit strategy and 'what next' if jejunal feeding fails. The evidence base for relief of functional symptoms (nausea and vomiting, early satiety, the feeling of fullness and pain) in the absence of a physical cause is limited. The authors discuss the multiple differential diagnosis including rumination syndrome, cyclical vomiting disorder, cannabinoid hyperemesis syndrome, functional dyspepsia, pseudoobstructive disorders, multisystem disorders (including neurological), disordered eating and food intolerance. There is a strong emphasis on a cohesive and rehabilitative focused multidisciplinary approach to improve outcomes avoiding jejunal feeding where possible. The authors discuss the concept of "effortful oral feeding as the least worst option" in the face of ongoing symptoms, in the absence of malnutrition, sometimes being the safest and most appropriate approach compared with the risks and complications associated with jejunal feeding (See page 397).

\section{Multi-professional in situ simulation is an effective method of identifying latent patient safety threats on the gastroenterology ward}

In situ simulation (ISS) is an effective training method for teams to deal with emergencies in high pressure environments. It can lead to improved patient safety by allowing the identification of latent patient safety threats. In this issue Uttley and colleagues report the 22 unannounced ISS sessions supervised by a trained faculty in a single centre over 2 years. The sessions were multi-professional (96 participants) and covered four common gastrointestinal emergencies - massive upper gastrointestinal bleeding, biliary sepsis, complications of endoscopic retrograde cholangiopancreatology and cardiac arrest. A full debrief followed each session - four main themes (systemic and human) were identified education and training, equipment, medication and team working. An action plan was put in place to deal with issues that had arisen including repeat testing. The detail is in the paper. Feedback from participants was very positive - $95 \%$ understood their role better, $86 \%$ felt more confident. $96 \%$ felt comfortable during the debrief. The process and its (ongoing) evaluation will, moving forward, potentially enable teams to improve efficiency and patient safety in a 'non-threatening' and constructive learning environment such as this - in effect learning on the job. There is an excellent accompanying commentary - the potential and challenges of utilising multi professional in situ simulation (See page 351 and Editor's Choice this month).

\section{Adult liver transplantation: a UK clinical guideline}

Liver transplantation is a highly successful treatment for all types of liver failure, some non-liver indications and liver cancer (up to $25 \%$ of liver transplants in the UK) with a 12 month survival in excess of $90 \%$. Although the transplant will be at one of the national centres management is across networks involving primary, secondary and tertiary care. In this issue we have published a multicentre evidence based two-part guidance specifically aimed at non-specialist clinicians caring for patients with acute and chronic liver disease. The first part examines: who to refer for liver transplant (LT), how to refer for LT, the LT assessment and how to manage the patient on the waiting list. The second part (surgery and post operation) covers organ donation, organ allocation and organ types, LT surgery and the post-operative period, immunosuppression, how the patient should be managed post-LT and Transplant outcomes. The authors incorporate recent national and international guidance where appropriate. Very practical and focussed - essential reading and well worth accessing as a resource (See page 375 and 385).

\section{Organisational changes and challenges for inflammatory bowel disease services in the UK during the COVID-19 pandemic}

The COVID-19 - 19 pandemic has presented significant challenges for patients with chronic medical disorders like inflammatory bowel disease (IBD) including the need to access healthcare despite services been diverted, the risk benefit of usual treatment and potential need for shielding. In this issue Kennedy and colleagues explore organisational changes made and challenges in delivering safe care - internet survey, 125 IBD units, April 2020. In summary there was a significant reduction in staffing parallel to a significant increase in IBD advice line contact with reduced access to face to face consultations, endoscopy and admissions. There was however an increased use of telemedicine, virtual meetings and noninvasive monitoring. There are several implications of this dataset from a period of uncertainty when rapid changes in how we practice and the evidence base that underpinned it emerged - the massive potential for morbidity in non COVID-19 patients, the 'reality' of diverting services but also potential new ways to deliver medical care during the ongoing pandemic and beyond (See page 343).

\section{Refeeding syndrome: physiological background and practical management}

Refeeding syndrome is the adverse clinical and biochemical state that can result from feeding malnourished patients via any route - oral, enteral or parenteral. In this issue De Silva and Nightingale review the physiological background and practical management. The main clinical problems relate to hypophosphataemia, hypomagnesia and hypokalaemia. Sodium retention is common. It is important to have a high index of suspicion especially in the malnourished patient who has had little or nothing to eat in the past 5 days. Generous electrolyte and vitamin replacement is essential. Non protein calories should be given as 50/50 carbohydrate and fat at $<50 \%$ requirements increased slowly with careful monitoring. Understanding the physiology is key to management. 


\section{UPFRONT}

The review is practical and focused. Well worth a read (See page 404).

\section{Finally}

The challenges of the COVID-19 pandemic continue and we have included in this edition a further review of recent papers (see page 341) which I hope is helpful.

Please continue to read, enjoy and feedback on the journal. Follow us on twitter @FrontGastro_BMJ and listen to our regular podcasts accessed via the journal website https://fg.bmj.com/

Keep safe and stay well.

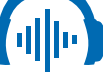

Listen to Podcast www.fg.bmj.com

ORCID iD

R Mark Beattie http://orcid.org/00000003-4721-0577 